\title{
Advances in Fuzzy Querying: Theory and Applications
}

\author{
Guy De Tré ${ }^{1}$, Janusz Kacprzyk², Adnan Yazici ${ }^{3}$, and Sławomir \\ Zadrożny ${ }^{2}$ \\ 1 Department of Telecommunications and Information Processing, Ghent University, \\ Sint-Pietersnieuwstraat 41, B-9000 Ghent, Belgium \\ Guy.DeTre@UGent.be \\ 2 Systems Research Institute, Polish Academy of Sciences, \\ ul. Newelska 6, 01-447 Warsaw, Poland \\ \{zadrozny, kacprzyk\} @ibspan. waw.pl \\ 3 Dept. of Computer Engineering, Middle East Technical University, \\ 06531, Ankara, Turkey \\ yazici@ceng.metu.edu.tr
}

\section{Preface}

Modern information systems, notably database management systems, are facing many challenges whose relevance and severity is constantly growing. These challenges are basically related to two aspects those systems are plagued of. The first is related to a sheer volume of data they have to deal with, and the second to a growing importance of the human factor in the sense that an overwhelming majority of users is novice, and yet they have to cope with huge data sets and sophisticated database technology. It is particularly true for all kinds of Web-based technologies, geographic information systems, multimedia systems, etc. This implies what might be called a 'human - database gap' the essence of which is that, on the one hand, limited and not growing human cognitive abilities are contrasted with rapidly growing sets of data and information/database system performance. On the other hand, a growing sophistication of data sets and problems faced by humans call for tools providing retrieval of information needed which should reflect sophisticated, often not precisely stated, intentions and preferences that are far apart of what traditional, precise and rigid querying languages may offer.

The purpose of this special issue is to collect new developments in that challenging field proposing more efficient and adequate data access, search and querying techniques which can bring about some breakthroughs for the above mentioned problems faced by the modern IT/ICT.

Soft computing and fuzzy set theory have been important tools in many research works that aim to deal with the intrinsic complexity of 
querying and information handling as required by current information systems. The aim of this special issue is to collect and present papers dealing with recent scientific advances in soft computing and fuzzy set theory, applied to flexible querying techniques.

This issue offers a selection of both theoretical and application oriented contributions, as a testimony and source of inspiration. As can be observed by studying them, not merely innovative ideas are thrown forward, but also well founded and advanced techniques, already being implemented in experimental environments are discussed.

The interest for advanced querying techniques, which are currently addressed separately by different research communities like databases, data warehouses, multimedia and decision support, is reflected in the related contributions selected for publication in this issue. In total, six papers have been included.

The first two papers are dealing with the handling of bipolarity in fuzzy database querying. In the paper by Matthé et al. bipolarity is reflected by positive and negative query criteria which respectively express what is satisfactory and what has to be rejected from the query results. In order to explicitly cope with the fact that positive and negative criteria do not necessarily have to complement each other, a novel bipolar query satisfaction modelling framework, which is based on an independent degree of satisfaction and degree of dissatisfaction, is studied. In the paper by Bosc and Pivert, a special case of bipolarity is considered. In this approach some criteria are considered to be mandatory and play the role of constraints, whereas satisfaction of other criteria is solely desirable. The authors then investigate how bipolarity may impact the division operator in the context of relational databases. Various forms of bipolar divisions are studied, each of them conveying specific semantics.

The next two papers deal with advanced multimedia search techniques. In the paper by Koyuncu it is studied how the query capabilities of multimedia databases can be extended through the integration of a fuzzy rule-based system. In addition to fuzzy semantic rules, which deduce new information from the data stored in the database, fuzzy spatial and temporal relations, which are inherent to multimedia applications, are defined in the rule-based system. If applicable, user queries then result in the deduction of new information using the rules defined in the rule-based system. In the paper by Doğan et al. the content-based classification and retrieval of real-world audio clips is studied. Four innovative flexible querying techniques for audio data are presented, namely, querying by mixed type audio classes, querying by domain based fuzzy classes, query- 
ing by temporal information and temporal relationships, and querying by example. A hash-based indexing technique is introduced to reduce retrieval time. The performance of the proposed system is evaluated by two kinds of experiments.

The next paper by Dujmovic and De Tré studies various fundamental aspects of multicriteria evaluation for land-use suitability analysis and the design of (geographic) suitability maps. Seven existing methods are investigated and compared from the standpoint of their ability to support the necessary logic properties that affect the expressive power of evaluation methods. Another contribution of the paper is the identification and evaluation of canonical forms of logic aggregation structures that can be used in the design of suitability maps. The practical use of these forms is illustrated by a method and a tool for making realistic and useful LSP suitability maps.

The last paper by Castillo et al. deals with data warehousing and linguistic summarisation in OLAP. More specifically, a methodology for providing linguistic answers to queries involving the comparison of time series obtained from data cubes with a time dimension is proposed. Time series related to events which are interesting for the user are obtained by querying data cubes using OLAP operations on the time dimension and summarised so that an appropriate short linguistic description is provided to the user. The approach is based on linguistically quantified statements and pointwise definitions of the degree and sign of local change.

We hope that the papers included in this issue will provide the reader with inspiration and motivation for the further development of interesting research field of fuzzy querying. Taking this opportunity we warmly invite all interested readers to visit the Web site of the EUSFLAT Working Group on Soft Computing in Database Management and Information Retrieval at http://scdmir.ugent.be. This special issue should be seen in part as an effect of the activities of this Working Group.

As guest editors of this special issue, we would like to express our gratitude to the authors for their contributions and the reviewers for their excellent cooperation. We sincerely thank Ron Yager, the Editor in Chief, for supporting us and giving us the opportunity of editing this issue.

Guy De Tré Janusz Kacprzyk

Adnan Yazici Sławomir Zadrożny 